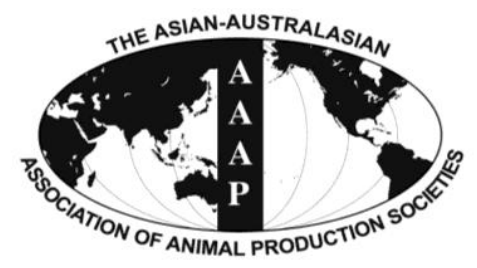

Asian Australas. J. Anim. Sci.

Vol. 26, No. 6 : 804-811 June 2013

http://dx.doi.org/10.5713/ajas.2012.12558

Www.ajas.info

pISSN $1011-2367$ elSSN 1976-5517

\title{
Effect of $\gamma$-Aminobutyric Acid (GABA) Producing Bacteria on In vitro Rumen Fermentation, Biogenic Amine Production and Anti-oxidation Using Corn Meal as Substrate
}

\author{
Bum Seung Ku, Lovelia L. Mamuad, Seon-Ho Kim, Chang Dae Jeong, Alvin P. Soriano, \\ Ho-Il Lee, Ki-Chang Nam ${ }^{1}$, Jong K. Ha ${ }^{2}$ and Sang Suk Lee* \\ Ruminant Nutrition and Anaerobe Laboratory, Department of Animal Science and Technology, \\ College of Bio-industry Science, Sunchon National University, Suncheon, Korea
}

\begin{abstract}
The effects and significance of $\gamma$-amino butyric acid (GABA) producing bacteria (GPB) on in vitro rumen fermentation and reduction of biogenic amines (histamine, methylamine, ethylamine, and tyramine) using corn meal as a substrate were determined. Ruminal samples collected from ruminally fistulated Holstein cows served as inoculum and corn was used as substrate at $2 \%$ dry matter (DM). Different inclusion rates of GPB and GABA were evaluated. After incubation, addition of GPB had no significant effect on in vitro fermentation $\mathrm{pH}$ and total gas production, but significantly increased the ammonia nitrogen $\left(\mathrm{NH}_{3}-\mathrm{N}\right)$ concentration and reduced the total biogenic amines production $(\mathrm{p}<0.05)$. Furthermore, antioxidation activity was improved as indicated by the significantly higher concentration of superoxide dismutase (SOD) and glutathione peroxidase (GSH-Px) among treated samples when compared to the control ( $\mathrm{p}<0.05$ ). Additionally, 0.2\% GPB was established as the optimum inclusion level. Taken together, these results suggest the potential of utilizing GPB as feed additives to improve growth performance in ruminants by reducing biogenic amines and increasing anti-oxidation. (Key Words: GABA, Biogenic Amines, Feed Additives, In vitro, Antioxidant)
\end{abstract}

\section{INTRODUCTION}

$\operatorname{GABA}(\gamma$-amino butyric acid), which is also known as 4-amino-butanoic acid, is a non-protein amino acid that acts as a major inhibitory neurotransmitter in the mammalian central nervous system. GABA is widely distributed in nature, been found in species ranging from microorganisms to plants and animals (Li et al., 2010), and is known for its multiple physiological functions including tranquilizing, diuretic and hypotensive activities (Jakobs et al., 1993; Inoue et al., 2003; Hayakawa et al., 2004). Furthermore, a number of studies have shown it has beneficial effects in animals such as etiotropic effects on the health status and

\footnotetext{
* Corresponding Author: Sang-Suk Lee. Tel: +82-61-750-3237, Fax: +82-61-750-3237, E-mail: rumen@sunchon.ac.kr

${ }^{1}$ Meat Science Laboratory, Department of Animal Science and Technology, College of Bio-industry Science, Sunchon National University, Suncheon, Korea.

2 Department of Agricultural Biotechnology and Research Institute for Agriculture and Life Sciences, College of Agriculture and Life Sciences, Seoul National University, Seoul, Korea. Submitted Oct. 9, 2012; Accepted Jan. 2, 2013; Revised Feb. 7, 2013
}

growth rates of calves (Matsumoto et al., 2009), capacitating action on ram spermatozoa (de las Heras et al., 1997), and protective effects against neurotoxicant-induced cell death (Cho et al., 2007).

Biogenic amines (BA) are generally formed through the decarboxylation of specific free amino acids by exogenous decarboxylases released by microbial species associated with fermented food and beverages. Ruminants potentially receive amines from both dietary and ruminal microbial sources and thus have the potential to absorb greater amounts than other species. Increased contents of dietary amines have been considered disadvantageous in ruminant nutrition since these substances tend to reduce dry matter intake (Van Os et al., 1995) and nitrogen degradability in the rumen of steers, particularly by the BA putrescine (Dawson and Mayne, 1997) and have negative effects on visceral organs and meat quality (Fusi et al., 2004).

The relationship between BA histamine concentration and hyperacidity during metabolic acidosis has also been reported in various functional studies of non-ruminant gastrointestinal cuticles (Miller et al., 1991; Groot Koerkamp et al., 1998; Komuro et al., 1998). However, this 
relationship has not been sufficiently investigated in the ruminant stomach (Underwood, 1992). Moreover, Aschenbach and Gäbel (2000) concluded that absorption of histamine leading to systemic acidosis is due to luminal acidity-induced ruminal epithelial damage and not histamine.

Stress factors such as high ambient temperature and humidity lead to loss of production by domestic animals (St-Pierre et al., 2003). To maintain homeostasis, animals respond to hot environments in various ways, including changes in capillary blood flow (Wolfenson et al., 1981) and respiration rate (Koelkebeck and Odom, 1994). Furthermore, decreased metabolism and adaptation of endocrine mechanisms also seem to play an important role in adaptation to hot environments (Beuving and Vonder, 1978).

GABA synthesis involves irreversible $\alpha$ decarboxylation of L-glutamic acid, a reaction catalyzed by glutamic acid decarboxylase (GAD) (Cho et al., 2007), which is found in various higher plants, animals, and bacteria (Ueno et al., 1997). However, studies in recent years focused primarily on GABA-producing lactic acid bacteria (LAB) because they possess special physiological activities, are generally regarded as safe, and enable safe and environmentally friendly production ( $\mathrm{Li}$ and Cao, 2010). LAB comprise an important group of gram-positive bacteria widely distributed in the environment that frequently exist in fermented food and vegetables and in the intestines of humans and animals (Omar et al., 2000; Gardner et al., 2001; Satokari et al., 2003). This study analyzed ruminal fermentation and content changes when different concentrations of the specific GPB isolate from kimchi, Lactobacillus brevis ATCC 14687, are added during in vitro fermentation and degradation of corn meal. Furthermore, the potential of GABA for reducing biogenic amine and increasing the level of the antioxidative substances superoxide dismutase (SOD) and glutathione peroxidase (GSH-Px) was investigated.

\section{MATERIALS AND METHODS}

\section{Experimental design and preparation of inoculum}

This study utilized different inclusion rates (v:v) of the $\gamma$-amino butyric acid (GABA) producing bacteria (GPB) Lactobacillus brevis YM 3-30 to investigate in vitro rumen fermentation using corn meal as a substrate. L. brevis was isolated from kimchi and anaerobically cultivated using MRS broth (Oxoid LTD, England) added with 5\% monosodium glutamate (MSG) at $32^{\circ} \mathrm{C}$, pH 5.0 for $48 \mathrm{~h}$. The treatments included 0 ppm GPB (T1), 2,000 ppm GPB, 5,000 ppm GPB (T3), 2,000 ppm autoclaved GPB (T4), 5,000 ppm autoclaved GPB (T5), 2,000 ppm GABA (T6), and 5,000 ppm GABA (T7). The GPB added had an optical density (OD) of 1.8 at $600 \mathrm{~nm}$. For GPB treatments, the added concentrations were based on the GABA produced by GBP. Additionally, the GPB was autoclaved at $121^{\circ} \mathrm{C}$ for 15 min prior to inclusion into the fermentation bottles for treatments 4 and 5. Commercially prepared GABA (SigmaAldrich Korea Ltd.) was used as the basis of comparison for treated samples (T6 and T7).

\section{In vitro experiment}

Rumen fluids were collected from fistulated Hanwoo cattle with body weights of $600 \pm 47 \mathrm{~kg}$. The collected fluids were filtered using cheese cloth that had been folded four times and placed in glass bottles. Samples of the pooled and particle-free rumen inoculum were placed in serum bottles, after which buffer medium containing different minerals was added in accordance with the method described by Russell and Van Soest (1984) to give a final mixture of $80 \%$ buffer and $20 \%$ rumen fluid with a $\mathrm{pH}$ of 6.7 . A constant $\mathrm{CO}_{2}$ gas flow was used to mix the rumen fluid and media to fill the serum bottles under anaerobic conditions. Fifty $\mathrm{ml}$ of buffered rumen fluid was anaerobically transferred to $160 \mathrm{ml}$ serum bottles containing $2 \%$ corn meal (DM). The filled serum bottles were then sealed with rubber stoppers and aluminum caps and incubated at $39^{\circ} \mathrm{C}$ and $100 \mathrm{rpm}$ for $0,12,24$ and $48 \mathrm{~h}$. Total gas (TG), $\mathrm{pH}$, ammonia nitrogen $\left(\mathrm{NH}_{3}-\mathrm{N}\right)$, volatile fatty acid (VFA), biogenic amines (BA), superoxide dismutase (SOD) and glutathione peroxidase (GSH-Px) were analyzed after incubation.

\section{Analyses for in vitro fermentation parameters}

TG production at different stages was measured in each of the serum bottles using a press and sensor machine (Laurel Electronics, Inc., Costa Mesa, CA, USA), while $\mathrm{pH}$ was measured with a Pinnacle series M530p meter (Schott instruments, Mainz, Germany) after uncapping each of the bottles. $\mathrm{NH}_{3}-\mathrm{N}$ concentration was measured according to the methods developed by Chaney and Marbach (1962). VFA and other metabolites were analyzed by high performance liquid chromatography (HPLC) (Agilent Technologies 1200 series, USA) according to the methods described by Tabaru et al. (1988) and Han et al. (2005).

BA concentrations were analyzed from the samples by high performance liquid chromatography (HPLC). Amine separation was conducted using HPLC (Waters Ltd., Massachusetts, USA) and a Varian column (Pursuit $\times$ Rs $5 \mathrm{u}$ C-18 250×4.6 mm) (Varian Inc., CA, USA). The fluorescence detector (Waters 470) was set to an excitation wavelength of $330 \mathrm{~nm}$ and an emission wavelength of 418 $\mathrm{nm}$. Amine compounds were identified and quantified based on comparison with standard curves constructed from pure compounds (Histamine, Methylamine, Ethylamine and 
Tyramine). The assay sensitivity for each amine was calculated from the extrapolation of a dilution curve and taken as the lowest concentration required to produce a signal-to-noise ratio of 3:1 (Snyder et al., 1997). The chromatographic data used was generated by the Mellenium32 System (Waters Millipore) program. HPLC samples were centrifuged at $16,609 \times \mathrm{g}$ for $5 \mathrm{~min}$ at $4^{\circ} \mathrm{C}$ and then filtered through $0.2 \mu \mathrm{m}$ millipore filters. Samples and $6 \mathrm{~N} \mathrm{HCl}$ were vortexed and hydrolyzed in an oven at $110^{\circ} \mathrm{C}$ for $24 \mathrm{~h}$, after which an Eyela SB-1000 evaporator (Tokyo Rikakikai Co. Ltd., Japan) was used to remove the HCl. The majority of the solutions used for HPLC were obtained from Sigma-Aldrich (Germany). Prior to sample analysis, a standard was developed between 0.98 and $1.0\left(\mathrm{r}^{2}\right)$.

\section{Antioxidant enzyme assays}

Total SOD activity was determined by the inhibition of pyrogallol autoxidation by SOD (with and without sample) at $25^{\circ} \mathrm{C}$ and was followed kinetically at $420 \mathrm{~nm}$ as described by Marklund and Marklund (1974). One unit of SOD is defined as the amount of enzyme that causes $50 \%$ inhibition of pyrogallol autoxidation. Changes in absorbance were read on a SPECTRAmax Plus microplate spectrophotometer (Molecular Devices Corp. Sunnyvale, CA, USA). Additionally, the GSH-Px was assayed at $25^{\circ} \mathrm{C}$ as described by Paglia and Valentine (1967) and LopezTorres et al. (1991), wherein NADPH oxidation was followed by spectrophotometry at $340 \mathrm{~nm}$ in the presence of reduced glutathione $(\mathrm{GSH})$ and hydrogen peroxide $\left(\mathrm{H}_{2} \mathrm{O}_{2}\right)$. To correct spontaneous reactions in the absence of enzyme, blanks were run without samples and then subtracted from the assay values. One unit of GSH-Px is defined as the amount of enzyme that oxidizes $1 \mathrm{mmol}$ of NADPH per minute. All enzyme activities were expressed in $\mathrm{U} / \mathrm{ml}$.

\section{Statistical analyses}

Data were analyzed by analysis of variance (ANOVA) using the general linear model (GLM) for a randomized complete block design. All treatments were conducted in triplicate and Duncan's Multiple Range Test (DMRT) was used to identify differences between specific treatments. A $\mathrm{p}<0.05$ was considered to indicate statistical significance. All analyses were carried out using Statistical Analysis Systems (SAS) version 9.1 (2002).

\section{RESULTS}

\section{pH, TG production and $\mathrm{NH}_{3}-\mathrm{N}$ concentration}

$\mathrm{pH}$, TG production and $\mathrm{NH}_{3}-\mathrm{N}$ concentration of in vitro fermentation at different incubation times using corn meal as a substrate are shown in Table $1 \mathrm{pH}$ decreased from 0 to $48 \mathrm{~h}$ of incubation; however, significant differences in $\mathrm{pH}$ among treatments were only observed during the first $12 \mathrm{~h}$ of incubation. Additionally, TG production and $\mathrm{NH}_{3}-\mathrm{N}$ concentration both increased with increased incubation time $(p<0.05)$. TG was found to be highest $(p<0.05)$ in T3, T4 and $\mathrm{T} 5$ after $24 \mathrm{~h}$ of incubation, while $\mathrm{NH}_{3}-\mathrm{N}$ concentration was lowest $(\mathrm{p}<0.05)$ in $\mathrm{T} 1 \quad(472.50 \mathrm{ppm})$ and highest $(\mathrm{p}<0.05)$ in T6 (585.33 ppm) and T7 (560.67 ppm) after 48 $\mathrm{h}$ of incubation.

\section{VFA production}

Table 2 shows the volatile fatty acid production (VFA) of in vitro fermentation amended with either GPB or GABA using cornmeal as a substrate. Although acetate production did not differ significantly, the highest value was observed

Table 1. $\mathrm{pH}$, total gas and ammonia nitrogen $\left(\mathrm{NH}_{3}-\mathrm{N}\right)$ concentration of in vitro fermentation amended with either GPB or GABA using cornmeal as substrate

\begin{tabular}{|c|c|c|c|c|c|c|c|c|c|c|}
\hline \multirow{2}{*}{ Time (h) } & \multicolumn{7}{|c|}{ Treatments } & \multirow{2}{*}{ Mean } & \multicolumn{2}{|c|}{ Linear } \\
\hline & $\mathrm{T} 1$ & $\mathrm{~T} 2$ & T3 & $\mathrm{T} 4$ & T5 & T6 & $\mathrm{T} 7$ & & Std error & p-value \\
\hline \multicolumn{11}{|l|}{$\overline{\mathrm{pH}}$} \\
\hline 0 & $5.90 \pm 0.01^{\mathrm{c}}$ & $6.01 \pm 0.02^{\mathrm{a}}$ & $5.96 \pm 0.01^{\mathrm{ab}}$ & $5.96 \pm 0.00^{\mathrm{ab}}$ & $5.96 \pm 0.01^{\mathrm{b}}$ & $5.96 \pm 0.02^{\mathrm{ab}}$ & $5.97 \pm 0.01^{\mathrm{ab}}$ & $5.96^{\mathrm{w}}$ & 0.06 & 0.0001 \\
\hline 12 & $5.32 \pm 0.01^{\mathrm{c}}$ & $5.32 \pm 0.01^{\mathrm{c}}$ & $5.39 \pm 0.01^{\mathrm{ab}}$ & $5.36 \pm 0.03^{\mathrm{bc}}$ & $5.42 \pm 0.02^{\mathrm{a}}$ & $5.32 \pm 0.01^{\mathrm{c}}$ & $5.37 \pm 0.01^{\mathrm{abc}}$ & $5.36^{\mathrm{x}}$ & & \\
\hline 24 & $5.04 \pm 0.08$ & $4.99 \pm 0.02$ & $5.01 \pm 0.03$ & $4.93 \pm 0.08$ & $5.06 \pm 0.04$ & $5.05 \pm 0.01$ & $5.00 \pm 0.04$ & $5.01^{\mathrm{y}}$ & & \\
\hline 48 & $4.83 \pm 0.11$ & $4.89 \pm 0.05$ & $4.92 \pm 0.07$ & $4.90 \pm 0.06$ & $4.96 \pm 0.01$ & $4.93 \pm 0.06$ & $4.93 \pm 0.05$ & $4.92^{\mathrm{z}}$ & & \\
\hline \multicolumn{11}{|c|}{ Total gas (ml) } \\
\hline 12 & $67.00 \pm 1.00$ & $65.00 \pm 1.15$ & $69.40 \pm 0.66$ & $66.00 \pm 3.60$ & $62.33 \pm 1.45$ & $67.33 \pm 0.66$ & $63.00 \pm 3.00$ & $65.71^{y}$ & 3.79 & 0.0001 \\
\hline 24 & $83.00 \pm 3.00^{\mathrm{bc}}$ & $77.67 \pm 2.18^{\mathrm{c}}$ & $85.67 \pm 1.33^{\mathrm{ab}}$ & $88.67 \pm 0.66^{\mathrm{a}}$ & $89.33^{\mathrm{a}} \pm 2.18$ & $78.33 \pm 1.45^{\mathrm{c}}$ & $80.67 \pm 1.20^{\mathrm{bc}}$ & $83.38^{\mathrm{x}}$ & & \\
\hline 48 & $92.50 \pm 0.50$ & $103.66 \pm 7.88$ & $100.66 \pm 3.71$ & $97.00 \pm 1.15$ & $92.33 \pm 2.33$ & $91.33 \pm 2.33$ & $99.00 \pm 5.77$ & $96.76^{\mathrm{w}}$ & & \\
\hline \multicolumn{11}{|c|}{$\mathrm{NH}_{3}-\mathrm{N}$ concentration (ppm) } \\
\hline 0 & $287.50 \pm 0.50^{\mathrm{b}}$ & $307.67 \pm 4.37^{\mathrm{ab}}$ & $332.00 \pm 4.72^{\mathrm{a}}$ & $327.33 \pm 0.33^{\mathrm{a}}$ & $331.67 \pm 8.68^{\mathrm{a}}$ & $308.67 \pm 6.33^{\mathrm{ab}}$ & $310.33 \pm 17.45^{\mathrm{ab}}$ & $311.11^{z}$ & 22.75 & 0.0001 \\
\hline 12 & $396.00 \pm 6.00^{\mathrm{a}}$ & $353.00 \pm 1.52^{\mathrm{b}}$ & $334.33 \pm 0.88^{\mathrm{b}}$ & $364.67 \pm 8.66^{\mathrm{ab}}$ & $327.00 \pm 1.73^{\mathrm{b}}$ & $324.00 \pm 1.15^{\mathrm{b}}$ & $336.33 \pm 31.39^{\mathrm{b}}$ & $347.60^{y}$ & & \\
\hline 24 & $411.50 \pm 0.50^{\mathrm{ab}}$ & $442.00 \pm 0.57^{\mathrm{a}}$ & $389.67 \pm 5.78^{\mathrm{bc}}$ & $412.33 \pm 1.20^{\mathrm{ab}}$ & $372.33 \pm 15.67^{\mathrm{c}}$ & $432.67 \pm 0.88^{\mathrm{a}}$ & $394.67 \pm 2.84^{\mathrm{b}}$ & $408.31^{x}$ & & \\
\hline 48 & $472.50 \pm 1.50^{\mathrm{d}}$ & $553.67 \pm 1.76^{\mathrm{b}}$ & $542.33 \pm 5.54^{\mathrm{bc}}$ & $523.00 \pm 5.85^{\mathrm{c}}$ & $554.67 \pm 1.20^{\mathrm{b}}$ & $585.33 \pm 3.17^{\mathrm{a}}$ & $560.67 \pm 18.55^{\mathrm{ab}}$ & $539.83^{w}$ & & \\
\hline
\end{tabular}

Means followed by the same superscript letter in a row $\left({ }^{\mathrm{a}, \mathrm{b}, \mathrm{c}, \mathrm{d}}\right)$ and column $\left({ }^{\mathrm{w}, \mathrm{x}, \mathrm{y}, \mathrm{z}}\right)$ are not significantly different at the $5 \%$ level by DMRT. T1: Control, T2: 2,000 ppm GPB, T3: 5,000 ppm GPB, T4: 2,000 ppm autoclaved GPB, T5: 5,000 ppm autoclaved GPB, T6: 2,000 ppm GABA chemical, T7: 5,000 ppm GABA chemical. 
Table 2. VFA production of in vitro fermentation amended with either GPB or GABA using cornmeal as substrate (ppm)

\begin{tabular}{|c|c|c|c|c|c|c|c|c|c|c|}
\hline \multirow{2}{*}{ Time (h) } & \multicolumn{7}{|c|}{ Treatments } & \multirow{2}{*}{ Mean } & \multicolumn{2}{|c|}{ Linear } \\
\hline & $\mathrm{T} 1$ & $\mathrm{~T} 2$ & T3 & $\mathrm{T} 4$ & T5 & T6 & $\mathrm{T} 7$ & & Std error & $\mathrm{p}$-value \\
\hline \multicolumn{11}{|l|}{ Acetate } \\
\hline 0 & $2,162.67 \pm 84.55^{\mathrm{a}}$ & $2,086.39 \pm 35.47^{\mathrm{a}}$ & $2,085.31 \pm 66.61^{\mathrm{a}}$ & $2,127.55 \pm 15.53^{\mathrm{a}}$ & $2,093.05 \pm 45.49^{\mathrm{a}}$ & $2,022.13 \pm 55.41^{\mathrm{ab}}$ & $1,915.83 \pm 28.24^{\mathrm{b}}$ & $2,070.42^{x}$ & 77.09 & 0.3820 \\
\hline 12 & $2,547.47 \pm 64.57$ & $2,417.87 \pm 59.60$ & $2,527.40 \pm 62.98$ & $2,395.11 \pm 38.26$ & $2,522.68 \pm 13.33$ & $2,483.44 \pm 26.87$ & $2,430.74 \pm 54.33$ & $2,474.95^{\mathrm{w}}$ & & \\
\hline 24 & $2,271.11 \pm 217.05$ & $2,620.63 \pm 76.20$ & $2,415.00 \pm 112.09$ & $2,315.00 \pm 112.09$ & $2,342.58 \pm 153.23$ & $2,447.48 \pm 59.09$ & $2,483.16 \pm 127.37$ & $2,413.56^{\mathrm{w}}$ & & \\
\hline 48 & $2,260.19 \pm 408.86$ & $2,618.01 \pm 135.97$ & $2,576.06 \pm 98.99$ & $2,419.31 \pm 112.77$ & $2,435.38 \pm 99.04$ & $2,416.50 \pm 96.59$ & $2,651.12 \pm 165.52$ & $2,482.36^{\mathrm{w}}$ & & \\
\hline \multicolumn{11}{|c|}{ Propionate } \\
\hline 0 & $632.15 \pm 1.90^{\mathrm{a}}$ & $576.16 \pm 3.54^{\mathrm{bcd}}$ & $563.53 \pm 1.49^{\mathrm{d}}$ & $577.77 \pm 4.60^{\mathrm{bc}}$ & $566.47 \pm 5.56^{\mathrm{cd}}$ & $585.47 \pm 2.70^{\mathrm{b}}$ & $573.07 \pm 4.18^{\mathrm{bcd}}$ & $582.08^{y}$ & 93.49 & 0.0240 \\
\hline 12 & $1,017.30 \pm 14.59^{\mathrm{abc}}$ & $894.25 \pm 26.01^{\mathrm{c}}$ & $970.63 \pm 46.62^{\mathrm{bc}}$ & $991.83 \pm 48.27^{\mathrm{abc}}$ & $1,117.42 \pm 30.80^{\mathrm{ab}}$ & $1,145.70 \pm 14.37^{\mathrm{a}}$ & $1,068.92 \pm 80.56^{\mathrm{ab}}$ & $1,029.43^{x}$ & & \\
\hline 24 & $1,195.14 \pm 156.39$ & $1,291.26 \pm 71.62$ & $1,292.37 \pm 4.15$ & $1,204.23 \pm 232.15$ & $1,403.70 \pm 32.64$ & $1,312.78 \pm 29.57$ & $1,354.44 \pm 35.04$ & $1,293.41^{x}$ & & \\
\hline 48 & $1,644.86 \pm 144.69$ & $1,543.34 \pm 235.48$ & $1,709.20 \pm 184.68$ & $1,971.53 \pm 65.46$ & $1,766.66 \pm 200.86$ & $1,759.23 \pm 145.66$ & $1,548.80 \pm 186.35$ & $1,706.23^{w}$ & & \\
\hline \multicolumn{11}{|l|}{ Butyrate } \\
\hline 0 & $427.27 \pm 3.76^{\mathrm{a}}$ & $385.58 \pm 11.64^{\mathrm{ab}}$ & $393.97 \pm 2.93^{\mathrm{ab}}$ & $380.12 \pm 32.41^{\mathrm{ab}}$ & $328.82 \pm 4.28^{\mathrm{b}}$ & $335.27 \pm 3.47^{\mathrm{b}}$ & $375.70 \pm 38.50^{\mathrm{ab}}$ & 375.28 & 92.44 & 0.0560 \\
\hline 12 & $906.65 \pm 23.09^{\mathrm{a}}$ & $751.65 \pm 66.19^{\mathrm{abc}}$ & $920.84 \pm 42.45^{\mathrm{a}}$ & $879.10 \pm 23.55^{\mathrm{a}}$ & $623.10 \pm 100.28^{\mathrm{c}}$ & $833.66 \pm 24.75^{\mathrm{ab}}$ & $685.04 \pm 41.08^{\mathrm{bc}}$ & 800.00 & & \\
\hline 24 & $1,187.85 \pm 112.54$ & $1,004.67 \pm 108.87$ & $1,290.89 \pm 151.00$ & $1,115.82 \pm 99.25$ & $1,415.50 \pm 259.96$ & $1,195.19 \pm 138.30$ & $1,067.96 \pm 101.32$ & $1,182.55$ & & \\
\hline 48 & $2,219.06 \pm 126.12$ & $2,609.40 \pm 207.83$ & $1,945.01 \pm 580.62$ & $1,903.84 \pm 71.65$ & $1,529.18 \pm 407.26$ & $1,551.95 \pm 407.26$ & $2,387.60 \pm 489.44$ & $2,020.86$ & & \\
\hline \multicolumn{11}{|l|}{ A:P ratio } \\
\hline 0 & $3.42 \pm 0.14^{\mathrm{ab}}$ & $3.62 \pm 0.07^{\mathrm{ab}}$ & $3.70 \pm 0.11^{\mathrm{a}}$ & $3.68 \pm 0.01^{\mathrm{a}}$ & $3.69 \pm 0.09^{\mathrm{a}}$ & $3.45 \pm 0.09^{\mathrm{ab}}$ & $3.34 \pm 0.05^{\mathrm{b}}$ & 3.55 & 0.53 & 0.0001 \\
\hline 12 & $2.50 \pm 0.02^{\mathrm{abc}}$ & $2.70 \pm 0.02^{\mathrm{a}}$ & $2.61 \pm 0.07^{\mathrm{ab}}$ & $2.42 \pm 0.08^{\mathrm{bc}}$ & $2.26 \pm 0.06^{\mathrm{cd}}$ & $2.17 \pm 0.02^{\mathrm{d}}$ & $2.29 \pm 0.13^{\mathrm{cd}}$ & 2.42 & & \\
\hline 24 & $1.91 \pm 0.07$ & $2.03 \pm 0.05$ & $1.87 \pm 0.03$ & $2.06 \pm 0.03$ & $1.67 \pm 0.08$ & $1.86 \pm 0.01$ & $1.83 \pm 0.05$ & 1.89 & & \\
\hline 48 & $1.55 \pm 0.02$ & $1.81 \pm 0.38$ & $1.56 \pm 0.25$ & $1.22 \pm 0.01$ & $1.43 \pm 0.24$ & $1.40 \pm 0.17$ & $1.79 \pm 0.34$ & 1.53 & & \\
\hline \multicolumn{11}{|c|}{ Total VFA } \\
\hline 0 & $3,222.09 \pm 86.41^{\mathrm{a}}$ & $3,048.12 \pm 43.47^{\mathrm{abc}}$ & $3,042.81 \pm 64.99^{\mathrm{abc}}$ & $3,085.44 \pm 49.46^{\mathrm{ab}}$ & $2,988.33 \pm 47.43^{\mathrm{bc}}$ & $2942.88 \pm 59.63^{\mathrm{bc}}$ & $2,864.54 \pm 58.06^{\mathrm{c}}$ & $3,027.74$ & 98.65 & 0.4730 \\
\hline 12 & $4,463.10 \pm 84.39$ & $4,264.60 \pm 94.82$ & $4,474.50 \pm 67.75$ & $4,361.40 \pm 104.87$ & $4,427.70 \pm 113.55$ & $4540.90 \pm 85.65$ & $4,365.90 \pm 26.81$ & $4,414.01$ & & \\
\hline 24 & $4,704.42 \pm 82.84^{\mathrm{c}}$ & $4,925.57 \pm 18.16^{\mathrm{b}}$ & $5,026.56 \pm 19.85^{\mathrm{b}}$ & $4,640.94 \pm 49.17^{\mathrm{c}}$ & $5,213.20 \pm 18.47^{\mathrm{a}}$ & $4932.49 \pm 64.29^{b}$ & $4,971.23 \pm 59.09^{\mathrm{b}}$ & $4,916.34$ & & \\
\hline 48 & $6,483.07 \pm 18.67^{\mathrm{b}}$ & $6,765.46 \pm 35.09^{\mathrm{a}}$ & $6,210.86 \pm 11.16^{\mathrm{c}}$ & $6,285.26 \pm 60.95^{\mathrm{c}}$ & $5,809.94 \pm 94.81^{\mathrm{d}}$ & $5768.41 \pm 45.52^{\mathrm{d}}$ & $6,618.17 \pm 16.98^{\mathrm{a}}$ & $6,277.31$ & & \\
\hline
\end{tabular}

Means followed by the same superscript letter in a row $\left({ }^{\mathrm{a}, \mathrm{b}, \mathrm{c}, \mathrm{d}}\right)$ and column $\left({ }^{\mathrm{w}, \mathrm{x}, \mathrm{y}, \mathrm{z}}\right)$ are not significantly different at the $5 \%$ level by DMRT.

T1: Control, T2: 2,000 ppm GPB, T3: 5,000 ppm GPB, T4: 2,000 ppm autoclaved GPB, T5: 5,000 ppm autoclaved GPB, T6: 2,000 ppm GABA chemical, T7: 5,000 ppm GABA chemical.

in T2 after $24 \mathrm{~h}$ of incubation and T2 and T7 after $48 \mathrm{~h}$ of incubation. Moreover, propionate production was highest in T5 (24 h) and T4 (48 h), while butyrate production was highest in T5 (24 h) and T2 (48 h). The acetate propionate ratio was highest in $\mathrm{T} 2$ and $\mathrm{T} 4$ after $24 \mathrm{~h}$ of incubation, while it was highest in $\mathrm{T} 2$ and $\mathrm{T} 7$ after $48 \mathrm{~h}$ incubation. Total VFA was highest $(p>0.05)$ in T5 after $24 \mathrm{~h}$ of incubation, while it was highest in $\mathrm{T} 2$ and $\mathrm{T} 7$ after $48 \mathrm{~h}$ of incubation.

\section{Biogenic amine analysis}

As shown in Table 3, only methylamine and ethylamine concentration values differed significantly between different incubation times, with the highest $(p<0.05)$ levels of both biogenic amines being observed at $48 \mathrm{~h}$. Among treatments, T1 showed the highest $(\mathrm{p}<0.05)$ amount of histamine production and total biogenic amines produced at 994.03 ppm and 1,042.29 ppm, respectively. Tyramine was found to be lower $(\mathrm{p}<0.05)$ in T2 and T3 after $24 \mathrm{~h}$ of incubation.

\section{Antioxidant enzyme assay}

As shown in Figure 1, the amounts of SOD and GSH-Px decreased $(\mathrm{p}<0.05)$ as the incubation period became longer. Additionally, the lowest $(\mathrm{p}<0.05)$ concentrations of SOD and GSH-Px of $80.36 \mathrm{U} / \mathrm{ml}$ and $27.67 \mathrm{U} / \mathrm{ml}$, respectively, were observed in T1 (control) when compared to treatments with GABA or GPB. Finally, the highest concentration of SOD $(90.50 \mathrm{U} / \mathrm{ml})$ was observed in $\mathrm{T} 7$, while that of GSH-
Px (33.87 U/ml) was observed in $\mathrm{T} 2$.

\section{DISCUSSION}

In vitro fermentation was conducted to simulate the rumen fermentation process. This method has been shown to be a valuable tool in various studies such as evaluation of the nutritive value of feedstuffs (Liu et al., 2002; Akinfemi et al., 2009; Murillo et al., 2011) and mitigation of methane production in ruminants (Do et al., 2011; Hassanat and Benchaar, 2012). The results of the present study indicated that, when cornmeal was used as the substrate, in vitro fermentation parameters became more positive with the addition of GPB and GABA chemicals, and $\mathrm{NH}_{3}-\mathrm{N}$ concentration, VFA production and biogenic amine concentration differed significantly from those of the control. Carbon dioxide was produced during the conversion of GABA into succinate and then propionate, which explains the increase in TG with the addition of chemical GABA and heat treated GBP. In addition, the increase of TG in T2 and T3 was due to the addition of live GBP, which increases the fermentation process and therefore TG production. Furthermore, involvement of the other pathway, the GABA to propionate conversion that leads to the production of carbon dioxide, also explains the increase of TG.

The addition of live L. brevis increases the microbial population and therefore the fermentation process; thus, $\mathrm{pH}$ 
Table 3. Biogenic amine concentration of in vitro fermentation amended with either GPB or GABA using cornmeal as substrate (ppm)

\begin{tabular}{|c|c|c|c|c|c|c|c|c|c|c|}
\hline \multirow{2}{*}{ Time (h) } & \multicolumn{7}{|c|}{ Treatments } & \multirow{2}{*}{ Mean } & \multicolumn{2}{|c|}{ Linear } \\
\hline & $\mathrm{T} 1$ & $\mathrm{~T} 2$ & $\mathrm{~T} 3$ & $\mathrm{~T} 4$ & $\mathrm{~T} 5$ & T6 & $\mathrm{T} 7$ & & $\begin{array}{l}\text { Std } \\
\text { error }\end{array}$ & $\mathrm{p}$-value \\
\hline \multicolumn{11}{|c|}{ Histamine } \\
\hline 0 & $636.23 \pm 23.47$ & $703.07 \pm 39.36$ & $698.70 \pm 43.20$ & $642.35 \pm 61.47$ & $661.32 \pm 34.63$ & $655.73 \pm 64.76$ & $641.20 \pm 42.72$ & 658.55 & 127.26 & 0.2080 \\
\hline 12 & $703.10 \pm 92.92^{\mathrm{ab}}$ & $561.20 \pm 13.61^{b}$ & $622.30 \pm 178.08^{\mathrm{ab}}$ & $764.70 \pm 32.35^{\mathrm{a}}$ & $816.40 \pm 119.50^{\mathrm{ab}}$ & $647.40 \pm 81.76^{\mathrm{ab}}$ & $591.10 \pm 26.14^{\mathrm{b}}$ & 737.25 & & \\
\hline 24 & $854.00 \pm 65.45^{\mathrm{a}}$ & $550.40 \pm 1.77^{\mathrm{b}}$ & $532.20 \pm 37.62^{\mathrm{b}}$ & $652.90 \pm 58.09^{\mathrm{ab}}$ & $837.90 \pm 166.43^{\mathrm{a}}$ & $580.90 \pm 27.71^{\mathrm{ab}}$ & $587.60 \pm 79.24^{\mathrm{ab}}$ & 667.47 & & \\
\hline 48 & $994.03 \pm 19.12^{\mathrm{a}}$ & $661.02 \pm 19.02^{\mathrm{b}}$ & $636.47 \pm 31.44^{\mathrm{b}}$ & $631.85 \pm 63.94^{\mathrm{b}}$ & $766.25 \pm 28.58^{\mathrm{ab}}$ & $602.52 \pm 19.60^{\mathrm{b}}$ & $565.78 \pm 15.80^{\mathrm{b}}$ & 679.20 & & \\
\hline \multicolumn{11}{|c|}{ Methylamine } \\
\hline 0 & $7.97 \pm 0.45$ & $7.56 \pm 0.57$ & $7.90 \pm 0.32$ & $7.27 \pm 0.13$ & $7.43 \pm 0.34$ & $7.48 \pm 0.50$ & $8.01 \pm 0.72$ & $7.64^{\mathrm{z}}$ & 0.98 & 0.0001 \\
\hline 12 & $8.71 \pm 0.04^{\mathrm{a}}$ & $8.79 \pm 0.42^{\mathrm{a}}$ & $8.67 \pm 0.21^{\mathrm{a}}$ & $8.23 \pm 0.14^{\mathrm{ab}}$ & $8.19 \pm 0.03^{\mathrm{ab}}$ & $8.36 \pm 0.08^{\mathrm{ab}}$ & $7.60 \pm 0.28^{b}$ & $8.39^{y}$ & & \\
\hline 24 & $9.36 \pm 0.36^{\mathrm{b}}$ & $9.42 \pm 0.27^{\mathrm{b}}$ & $11.32 \pm 1.27^{\mathrm{a}}$ & $9.41 \pm 0.27^{\mathrm{b}}$ & $9.16 \pm 0.06^{\mathrm{b}}$ & $9.29 \pm 0.14^{\mathrm{b}}$ & $9.22 \pm 0.03^{\mathrm{b}}$ & $9.58^{x}$ & & \\
\hline 48 & $12.90 \pm 1.85$ & $12.22 \pm 0.85$ & $13.34 \pm 1.74$ & $11.21 \pm 0.11$ & $12.04 \pm 0.58$ & $11.07 \pm 0.07$ & $11.16 \pm 0.06$ & $11.99^{\mathrm{w}}$ & & \\
\hline \multicolumn{11}{|c|}{ Ethylamine } \\
\hline 0 & $15.61 \pm 1.41^{\mathrm{a}}$ & $14.00 \pm 1.21^{\mathrm{ab}}$ & $11.70 \pm 0.20^{\mathrm{b}}$ & $12.82 \pm 0.86^{\mathrm{b}}$ & $12.75 \pm 0.38^{\mathrm{b}}$ & $13.36 \pm 0.91^{\mathrm{ab}}$ & $12.26 \pm 0.48^{\mathrm{b}}$ & $13.05^{y}$ & 6.40 & 0.0001 \\
\hline 12 & $12.97 \pm 0.37^{\mathrm{c}}$ & $17.81 \pm 0.30^{\mathrm{a}}$ & $15.32 \pm 0.77^{\mathrm{b}}$ & $17.48 \pm 1.23^{\mathrm{a}}$ & $14.57 \pm 0.22^{\mathrm{bc}}$ & $15.10 \pm 0.22^{\mathrm{b}}$ & $14.03 \pm 0.27^{\mathrm{bc}}$ & $15.59^{x y}$ & & \\
\hline 24 & $15.80 \pm 0.07$ & $16.02 \pm 0.33$ & $18.41 \pm 2.27$ & $17.72 \pm 1.21$ & $23.03 \pm 7.14$ & $15.73 \pm 0.08$ & $16.84 \pm 0.41$ & $17.65^{\mathrm{x}}$ & & \\
\hline 48 & $26.09 \pm 0.06^{\mathrm{b}}$ & $30.83 \pm 8.04^{\mathrm{b}}$ & $48.94 \pm 7.51^{\mathrm{a}}$ & $27.44 \pm 6.47^{\mathrm{b}}$ & $21.80 \pm 3.12^{\mathrm{b}}$ & $19.33 \pm 1.10^{\mathrm{b}}$ & $21.63 \pm 3.97^{\mathrm{b}}$ & $27.91^{\mathrm{w}}$ & & \\
\hline \multicolumn{11}{|l|}{ Tyramine } \\
\hline 0 & $47.17 \pm 1.93^{\mathrm{ab}}$ & $37.98 \pm 2.63^{\mathrm{b}}$ & $40.74 \pm 8.44^{\mathrm{ab}}$ & $40.30 \pm 3.89^{\mathrm{ab}}$ & $55.21 \pm 4.22^{\mathrm{a}}$ & $54.02 \pm 2.48^{\mathrm{a}}$ & $47.76 \pm 2.70^{\mathrm{ab}}$ & 46.68 & 7.39 & 0.4100 \\
\hline 12 & $30.24 \pm 1.02^{\mathrm{c}}$ & $45.29 \pm 3.05^{\mathrm{ab}}$ & $36.01 \pm 5.45^{\mathrm{bc}}$ & $48.35 \pm 1.92^{\mathrm{a}}$ & $49.51 \pm 2.27^{\mathrm{a}}$ & $45.26 \pm 2.45^{\mathrm{ab}}$ & $45.32 \pm 0.62^{\mathrm{ab}}$ & 42.83 & & \\
\hline 24 & $41.42 \pm 1.36^{\mathrm{ab}}$ & $34.87 \pm 1.29^{\mathrm{b}}$ & $32.80 \pm 0.62^{\mathrm{b}}$ & $50.08 \pm 3.71^{\mathrm{a}}$ & $51.45 \pm 2.18^{\mathrm{a}}$ & $53.75 \pm 1.54^{\mathrm{a}}$ & $50.17 \pm 8.55^{\mathrm{a}}$ & 44.91 & & \\
\hline 48 & $46.22 \pm 4.53$ & $46.32 \pm 1.35$ & $42.37 \pm 3.54$ & $43.04 \pm 4.11$ & $47.52 \pm 5.11$ & $43.28 \pm 7.35$ & $47.32 \pm 1.97$ & 45.62 & & \\
\hline \multicolumn{11}{|c|}{ Total biogenic amine } \\
\hline 0 & $706.97 \pm 27.26$ & $762.61 \pm 38.74$ & $759.04 \pm 35.18$ & $702.73 \pm 64.28$ & $736.70 \pm 38.89$ & $730.60 \pm 67.95$ & $709.23 \pm 42.58$ & 725.91 & 129.78 & 0.1900 \\
\hline 12 & $770.00 \pm 79.36^{\mathrm{ab}}$ & $633.10 \pm 16.55^{\mathrm{b}}$ & $691.70 \pm 176.83^{\mathrm{b}}$ & $838.70 \pm 33.70^{\mathrm{ab}}$ & $903.40 \pm 124.58^{\mathrm{a}}$ & $735.30 \pm 91.12^{\mathrm{ab}}$ & $662.70 \pm 26.24^{\mathrm{b}}$ & 811.49 & & \\
\hline 24 & $936.60 \pm 67.08^{\mathrm{a}}$ & $625.50 \pm 6.81^{\mathrm{b}}$ & $594.40 \pm 29.53^{\mathrm{ab}}$ & $747.10 \pm 58.17^{\mathrm{ab}}$ & $925.70 \pm 177.84^{\mathrm{a}}$ & $672.40 \pm 28.97^{\mathrm{ab}}$ & $663.90 \pm 70.55^{\mathrm{ab}}$ & 754.36 & & \\
\hline 48 & $1,042.29 \pm 10.12^{\mathrm{a}}$ & $774.65 \pm 32.59^{b}$ & $781.02 \pm 36.78^{b}$ & $766.03 \pm 59.36^{\mathrm{b}}$ & $755.06 \pm 33.95^{\mathrm{b}}$ & $709.88 \pm 22.08^{\mathrm{b}}$ & $722.10 \pm 18.56^{\mathrm{b}}$ & 785.33 & & \\
\hline
\end{tabular}

Means followed by the same superscript letter in a row $\left({ }^{\mathrm{a}, \mathrm{b}, \mathrm{c}, \mathrm{d}}\right)$ and column $\left({ }^{\mathrm{w}, \mathrm{x}, \mathrm{y}, \mathrm{z}}\right)$ are not significantly different at the $5 \%$ level by DMRT.

T1: Control, T2: 2,000 ppm GPB, T3: 5,000 ppm GPB, T4: 2,000 ppm autoclaved GPB, T5: 5,000 ppm autoclaved GPB, T6: 2,000 ppm GABA chemical, T7: 5,000 ppm GABA chemical.

decreased as a result of lactic acid production by L. brevis. addition of L. brevis should lead to the accumulation of Russell et al. (1979) found that extending the feed lactic acid. If the $\mathrm{pH}$ value decreases below 6.2, low fiber fermentation time using soluble carbohydrates caused a digestion will occur due to a reduction in fiber degrading decrease in $\mathrm{pH}$ value due to the rapid production of lactic bacteria. However, there were no significant differences acid in ruminants. Using corn meal as the substrate and observed in the mean values of $\mathrm{pH}$. These findings may

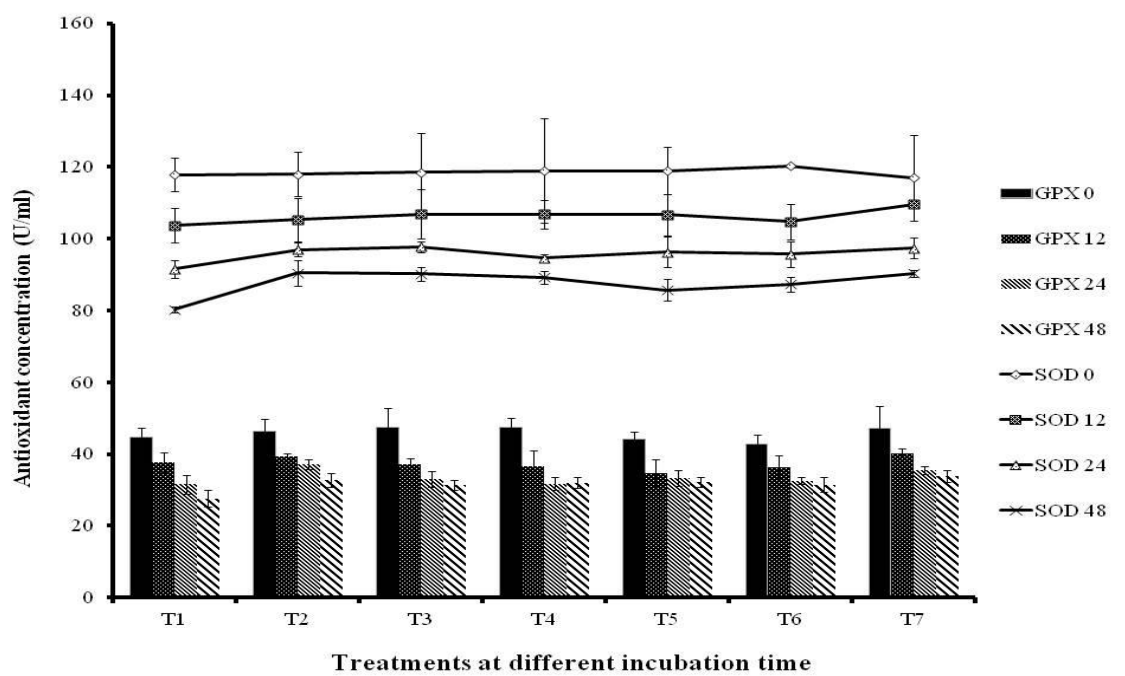

Figure 1. Antioxidant concentration of in vitro fermentation amended with either GPB or GABA using cornmeal as substrate (U/ml). Values are the means of triplicate analyses and bars indicate the standard error. Treatments: T1: Control, T2: 2,000 ppm GPB, T3: 5,000 ppm GPB, T4: 2,000 ppm autoclaved GPB, T5: 5,000 ppm autoclaved GPB, T6: 2,000 ppm GABA chemical, T7: 5,000 ppm GABA chemical. 
have been due to GABA production of $L$. brevis and the addition of external GABA. GABA might inhibit histamine production, which is responsible for stimulating $\mathrm{HCl}$ secretion in the stomach through histamine $\mathrm{H}_{2}$ receptors. The apparently comparable $\mathrm{pH}$ values between the control and treated groups suggest that the addition of GPB (live and heat treated) and GABA did not make the in vitro fermentation more acidic; hence, their addition is considered advantageous.

The maximum growth of rumen microbes is considered to occur under a concentration of 2 to $5 \mathrm{mg} \mathrm{NH} \mathrm{NH}_{3} \mathrm{~N}$ per 100 $\mathrm{ml}$ of rumen fluid (Slyter et al., 1979), and a minimum of about $80 \mathrm{mg} \mathrm{N}$ per liter is required to achieve the maximum degradation of carbohydrates by rumen microbes (Dryhurst and Wood, 1998). However, the $\mathrm{NH}_{3}-\mathrm{N}$ concentration in the ruminant stomach is between 1 to $76 \mathrm{mg} / 100 \mathrm{ml}$ of rumen fluids (Syrjålå et al., 1973). GPB (live and heat treated) and chemical GABA are sources of protein in the rumen. Hence, higher amounts of $\mathrm{NH}_{3}-\mathrm{N}$ were observed in the treated groups than the control, but still in its range of $\mathrm{NH}_{3}-\mathrm{N}$ concentration in the ruminant stomach. This observation might have been due to the use of corn meal as the substrate, which is a source of carbohydrate. Russell et al. (1983) found that when carbohydrate availability increases, ammonia production decreases because of direct incorporation of amino $\mathrm{N}$ into microbial protein, thus bypassing the ammonia pool.

Biogenic amines are present in all natural products in which protein degradation has occurred. Tyramine, which is an indirect sympathomimetic, can cause a hypertensive reaction and has been found to be associated with increased blood pressures and headaches. Moreover, Shalaby (1996) reported that tyramine and histamine can evoke unwanted symptoms such as nausea, vomiting, migraine, hypertension and headache. Furthermore, methylamine, which is also a BA, is used as a substrate for methanogenesis (Thauer, 1998). Costantini et al. (2009) showed that species of Lactobacillus were able to produce histamine. Conversely, Huang et al. (2007) used L. brevis for the production of GABA on an industrial scale. The apparent lower concentration of histamine, tyramine and TBA in the treated groups than the control indicates a positive effect of GPB (live or heat treated) and chemical GABA inclusion. Thus, L. brevis, which we previously isolated and found to produce GABA, might inhibit or regulate certain BA compounds like histamine and tyramine.

Glutamate is an excitatory amino acid found in all foods containing protein that is synthesized from glutamine by the enzyme glutaminase. There are several sources of protein in the rumen, one of which is microbial protein that may be the source for glutamate production. Glutamate decarboxylase or glutamic acid decarboxylase (GAD) is an enzyme that catalyzes the decarboxylation of glutamate to
GABA and $\mathrm{CO}_{2}$. Through the GABA shunt, GABA transaminase enzyme catalyzes the conversion of GABA and 2-oxoglutarate into succinic semialdehyde and glutamate, respectively. Succinic semialdehyde is then oxidized into succinic acid by succinic semialdehyde dehydrogenase and then enters the citric acid cycle as a usable source of energy (Shelp et al., 1999). Propionate can be produced from succinate through succinate dehydrogenase. This pathway explains the increase of propionate in treatments amended with GBP (live or heat treated) and chemical GABA, especially in T4 and T5.

Ruminants can be exposed to toxic concentrations of different environmental pollutants, including heavy metals, by consumption of contaminated feed and water (Holovská et al., 2002). The ingested toxic substances can be inhibited to both the fermentative activity and growth of the microbes, thereby changing the physiological steady state of the rumen fermentation (Javorsky et al., 1993; Lenártová et al., 1998). The pollutants can also contribute to enhancing the free oxyradicals content, which could generate oxidative and environmental stress for ruminal microbes. Microorganisms have developed efficient enzymatic and nonenzymatic mechanisms to eliminate these toxic and mutagenic oxygen by-products (Storz et al., 1990). The addition of the GPB significantly improves antioxidant activity by increasing SOD and GSH-Px, which is consistent with the results of experiments conducted in rats (Baydas et al., 2005). These substances are the primary oxygen free radical eliminators, which decrease during stressful environmental conditions. An investigation of the evaluation of the anti-inflammatory effects of catalase (CAT) or SOD producing $\mathrm{LAB}$ on mice using a trinitrobenzenesulfonic acid (TNBS) induced Crohn's disease murine model showed that mice administered CAT or SOD-producing LAB had a faster recovery of initial weight loss, increased enzymatic activities in the gut and lesser intestinal inflammation than animals that did not receive bacterial supplementation SOD producing LAB (LeBlanc et al., 2011). Additionally, improvement in the antioxidation in response to significant increases in the activity of SOD and GSH-Px was observed by Zhang et al. (2012) in experiments in laying hens subjected to heat stress.

\section{CONCLUSIONS}

The result of the present study indicates the potential use of GPB in the development of feed additives. Inclusion at 2,000 ppm gave the optimum result of reducing biogenic amine and increasing the $\mathrm{NH}_{3}-\mathrm{N}$ production and antioxidant activity under the in vitro fermentation technique. However, the possible role of GPB in increasing $\mathrm{NH}_{3}-\mathrm{N}$ concentration and antioxidants SOD and GSH-Px and reducing biogenic amine production in the treated samples has yet to be 
established. Their interactions with rumen microorganisms should also be studied to understand how GBP induces probiotic effects in cattle. Furthermore, in vivo studies should be conducted to determine the actual effects of utilizing GPB to improve the performance of ruminants.

\section{ACKNOWGLEDGEMENT}

This research was supported by the Technology Development Program for Agriculture and Forestry, Ministry of Agriculture, Forestry and Fisheries, Republic of Korea, 2012. The project was titled as "Development of production technologies for high quality and nutritional values of beef in Hanwoo"

\section{REFERENCES}

Akinfemi, A., A. O. Adesanya and V. E. Aya. 2009. Use of an in vitro gas production technique to evaluate some Nigerian feedstuffs. Am. Eur. J. Sci. Res. 4:240-245.

Aschenbach, J. R. and G. Gäbel. 2000. Effect and absorption of histamine in sheep rumen: Significance of acidotic epithelial damage. J. Anim. Sci. 78:464-470.

Baydas, G., F. Ozveren, I. Akdemir, M. Tuzcu and A. Yasar. 2005. Learning and memory deficits in rats induced by chronic thinner exposure are reversed by melatonin. J. Pineal. Res. 39:50-56

Beuving, G. and G. M. Vonder. 1978. Effect of stressing factors on corticosterone levels in the plasma of laying hens. Gen. Comp. Endocrinol. 35:153-159.

Cho, Y. R., J. Y. Chang and H. C. Chang. 2007. Production of gamma-aminobutyric acid (GABA) by Lactobacillus buchneri isolated from kimchi and its neuroprotective effect on neuronal cells. J. Microbiol. Biotechnol. 17:104-109.

Costantini, A., E. Vaudano, V. Del Prete, M. Danei and E. GarciaMoruno. 2009. Biogenic amine production by contaminating bacteria found in starter preparations used in winemaking. J. Agric. Food Chem. 57:10664-10669.

Dawson, L. E. R. and C. S. Mayne. 1997. The effect of infusion of putrescine and gamma amino butyric acid on the intake of steers offered grass silage containing three levels of lactic acid. Anim. Feed Sci. Technol. 66:15-29.

de las Heras, M. A., A. Valcarcel and L. J. Perez. 1997. In vitro capacitating effect of gamma-aminobutyric acid in ram spermatozoa. Biol. Reprod. 56:964-968.

Do, H. Q., T. Thi Thuy, T. Phuc Hao, T. R. Preston and R. A. Leng. 2011. Effects of nitrate and sulphur on in vitro methane production and dry matter degradation. Livest. Res. Rural Dev. 23.

Dryhurst, N. and C. D. Wood. 1998. The effect of nitrogen source and concentration on in vitro gas production using rumen micro-organisms. Anim. Feed Sci. Technol. 71:131-143.

Fusi, E., L. Rossi, R. Rebucci, F. Cheli, A. Di Giancamillo, C. Domeneghini, L. Pinotti, V. Dell'Orto and A. Baldi. 2004. Administration of biogenic amines to Saanen kids: effects on growth performance, meat quality and gut histology. Small
Rumin. Res. 53:1-7.

Gardner, N. J., T. Savard, P. Obermeier, G. Caldwell and C. P. Champagne. 2001. Selection and characterization of mixed starter cultures for lactic acid fermentation of carrot, cabbage, beet and onion vegetable mixtures. Int. J. Food Microbiol. 64:261-275.

Groot Koerkamp, P. W. G., J. H. M. Metz, G. H. Uenk, V. R. Phillips, M. R. Holden, R. W. Sneath, J. L. Short, R. P. White, J. Hartung, J. Seedorf, M. Schröder, K. H. Linkertc, S. Pedersend, H. Takaid, J. O. Johnsend and C. M. Wathes. 1998. Concentrations and emissions of ammonia in livestock buildings in Northern Europe. J. Agric. Eng. Res. 70:79-95.

Han, S.-K., S.-H. Kim and H.-S. Shin. 2005. UASB treatment of wastewater with VFA and alcohol generated during hydrogen fermentation of food waste. Process Biochem. 40:2897-2905.

Hassanat, F. and C. Benchaar. 2012. Assessment of the effect of condensed (acacia and quebracho) and hydrolysable (chestnut and valonea) tannins on rumen fermentation and methane production in vitro. J. Sci. Food Agric. 93:332-339.

Hayakawa, K., M. Kimura, K. Kasaha, K. Matsumoto, H. Sansawa and Y. Yamori. 2004. Effect of a gamma-aminobutyric acidenriched dairy product on the blood pressure of spontaneously hypertensive and normotensive Wistar-Kyoto rats. Br. J. Nutr. 92:411-417.

Holovská, K., V. Lenártová, P. Pristaš and P. Javorský. 2002. Are ruminal bacteria protected against environmental stress by plant antioxidants? Lett. Appl. Microbiol. 35:301-304.

Huang, J., L.-h. Mei, H. Wu and D.-q. Lin. 2007. Biosynthesis of $\gamma$-aminobutyric acid (GABA) using immobilized whole cells of Lactobacillus brevis. World J. Microbiol. Biotechnol. 23:865871.

Inoue, K., T. Shirai, H. Ochiai, M. Kasao, K. Hayakawa, M. Kimura and H. Sansawa. 2003. Blood-pressure-lowering effect of a novel fermented milk containing gamma-aminobutyric acid (GABA) in mild hypertensives. Eur. J. Clin. Nutr. 57:490495

Jakobs, C., J. Jaeken and K. M. Gibson. 1993. Inherited disorders of GABA metabolism. J. Inherit. Metab. Dis. 16:704-715.

Javorsky, P., E. Rybosova, Z. Mayerova, H. Mlynarcikova and J. Legath. 1993. Resistance of ruminal microorganisms to supermethrin. Vet. Hum. Toxicol. 35:15-18.

Koelkebeck, K. W. and T. W. Odom. 1994. Laying hen responses to acute heat stress and carbon dioxide supplementation: I. Blood gas changes and plasma lactate accumulation. Comp. Biochem. Physiol. Comp. Physiol. 107:603-606.

Komuro, Y., K. Ishihara, Y. Kojima, K. Saigenji and K. Hotta. 1998. Distinct effects of tetragastrin in rat gastroduodenal mucosa on mucin content and mucosal protective action against histamine-induced injury. Dig. Dis. Sci. 43:1050-1056.

LeBlanc, J. G., S. del Carmen, A. Miyoshi, V. Azevedo, F. Sesma, P. Langella, L. G. Bermúdez-Humarán, L. Watterlot, G. Perdigon and A. de Moreno de LeBlanc. 2011. Use of superoxide dismutase and catalase producing lactic acid bacteria in TNBS induced Crohn's disease in mice. J. Biotechnol. 151:287-293.

Lenártová, V., K. Holovská and P. Javorský. 1998. The influence of mercury on the antioxidant enzyme activity of rumen bacteria Streptococcus bovis and Selenomonas ruminantium. 
FEMS Microbiol. Ecol. 27:319-325.

Li, H. and Y. Cao. 2010. Lactic acid bacterial cell factories for gamma-aminobutyric acid. Amino Acids 39:1107-1116.

Li, H., T. Qiu, G. Huang and Y. Cao. 2010. Production of gammaaminobutyric acid by Lactobacillus brevis NCL912 using fedbatch fermentation. Microb. Cell Fact. 9:85.

Liu, J. X., A. Susenbeth and K. H. Sudekum. 2002. In vitro gas production measurements to evaluate interactions between untreated and chemically treated rice straws, grass hay, and mulberry leaves. J. Anim. Sci. 80:517-524.

Lopez-Torres, M., R. Perez-Campo and G. Barja de Quiroga. 1991. Aging in brown fat: antioxidant defenses and oxidative stress. Mech. Ageing Dev. 59:129-137.

Marklund, S. and G. Marklund. 1974. Involvement of the superoxide anion radical in the autoxidation of pyrogallol and a convenient assay for superoxide dismutase. Eur. J. Biochem. 47:469-474.

Matsumoto, D., M. Takagi, Y. Fushimi, K. Okamoto, M. Kido, M. Ryuno, Y. Imura, M. Matsunaga, K. Inokoshi, F. Shahada and E. Deguchi. 2009. Effects of gamma-aminobutyric acid administration on health and growth rate of group-housed Japanese black calves fed using an automatic controlled milk feeder. J. Vet. Med. Sci. 71:651-656.

Miller, M. J., X. J. Zhang, B. Barkemeyer, H. Sadowska-Krowicka, S. Eloby-Childress, X. Gu and D. A. Clark. 1991. Potential role of histamine monochloramine in a rabbit model of ileitis. Scand. J. Gastroenterol. 26:852-858.

Murillo, M., E. Herrera, O. Reyes, J. N. Gurrola and E. Gutierrez. 2011. Use in vitro gas production technique for assessment of nutritional quality of diets by range steers. Afr. J. Agric. Res. 6:2522-2526.

Omar, N. B., F. Ampe, M. Raimbault, J. P. Guyot and P. Tailliez. 2000. Molecular diversity of lactic acid bacteria from cassava sour starch (Colombia). Syst. Appl. Microbiol. 23:285-291.

Paglia, D. E. and W. N. Valentine. 1967. Studies on the quantitative and qualitative characterization of erythrocyte glutathione peroxidase. J. Lab. Clin. Med. 70:158-169.

Russell, J. B., W. M. Sharp and R. L. Baldwin. 1979. The effect of $\mathrm{pH}$ on maximum bacterial growth rate and its possible role as a determinant of bacterial competition in the rumen. J. Anim. Sci. 48:251-255.

Russell, J. B., C. J. Sniffen and P. J. Van Soest. 1983. Effect of carbohydrate limitation on degradation and utilization of casein by mixed rumen bacteria. J. Dairy Sci. 66:763-775.

Russell, J. B. and P. J. Van Soest. 1984. In vitro ruminal fermentation of organic acids common in forage. Appl. Environ. Microbiol. 47:155-159.

SAS. 2002. SAS/STAT. Statistical Analysis Systems for Windows. Release 9.1. SAS Institute Inc., Cary, NC, USA.
Satokari, R. M., E. E. Vaughan, H. Smidt, M. Saarela, J. Matto and W. M. de Vos. 2003. Molecular approaches for the detection and identification of Bifidobacteria and Lactobacilli in the human gastrointestinal tract. Syst. Appl. Microbiol. 26:572584.

Shalaby, A. R. 1996. Significance of biogenic amines to food safety and human health. Food Res. Int. 29:675-690.

Shelp, B. J., A. W. Bown and M. D. McLean. 1999. Metabolism and functions of gamma-aminobutyric acid. Trends Plant Sci. 4:446-452.

Slyter, L. L., L. D. Satter and D. A. Dinius. 1979. Effect of ruminal ammonia concentration on nitrogen utilization by steers. J Anim. Sci. 48:906-912.

Snyder, L. R., J. J. Kirkland and J. L. Glajch. 1997. Practical HPLC Method Development. John Wiley and Sons, New York, USA. pp. 180.

St-Pierre, N. R., B. Cobanov and G. Schnitkey. 2003. Economic losses from heat stress by US livestock industries. J. Dairy Sci. 86:E52-E77.

Storz, G., L. A. Tartaglia, S. B. Farr and B. N. Ames. 1990. Bacterial defenses against oxidative stress. Trends Genet. 6:363-368.

Syrjålå, L., V. Kossila and H. Sipila. 1973. A study of nutritional status of Finnish reindeer (Rangifer tarandus L.) in different months. I. Composition and volume of the microbiota. J. Sci. Agric. Soc. Finl. 45:534-541.

Tabaru, H., E. Kadota, H. Yamada, N. Sasaki and A. Takeuchi. 1988. Determination of volatile fatty acids and lactic acid in bovine plasma and ruminal fluid by high performance liquid chromatography. Jpn. J. Vet. Sci. 50:1124-1126.

Thauer, R. K. 1998. Biochemistry of methanogenesis: a tribute to Marjory Stephenson. 1998 Marjory Stephenson Prize Lecture. Microbiology 144:2377-2406.

Ueno, Y., K. Hayakawa, S. Takahashi and K. Oda. 1997. Purification and characterization of glutamate decarboxylase from Lactobacillus brevis IFO 12005. Biosci. Biotechnol. Biochem. 61:1168-1171.

Underwood, W. J. 1992. Rumen lactic acidosis. Part 2. Clinical signs, diagnosis, treatment and prevention. Compend. Cont. Educ. Pract. Vet. 14:1265-1270.

Van Os, M., J. P. Dulphy and R. Baumont. 1995. The effect of protein degradation products in grass silages on feed intake and intake behaviour in sheep. Br. J. Nutr. 73:51-64.

Wolfenson, D., Y. Frei, N. Snapir and A. Berman. 1981. Heat stress effects on capillary blood flow and its redistribution in the laying hen. Pflugers. Arch. 390:86-93.

Zhang, M., X. T. Zou, H. Li, X. Y. Dong and W. Zhao. 2012. Effect of dietary gamma-aminobutyric acid on laying performance, egg quality, immune activity and endocrine hormone in heat-stressed Roman hens. Anim. Sci. J. 83:141147. 